\title{
Desenvolvimento vegetativo e produção de óleo essencial de patchouli, sombreamento e aplicação de $\mathbf{G A}_{3}$
}

\section{Vegetative development and production of essential oil of Patchouli under radiation levels and $\mathrm{GA}_{3}$ application}

\author{
Rafaellen Caroline Storck ${ }^{1 *}$; Cícero Deschamps ${ }^{2}$; Lílian Cristina Côcco ${ }^{3}$; \\ Átila Francisco Mógor ${ }^{2}$; Agnes de Paula Scheer ${ }^{4}$; Carlos Itsuo Yamamoto ${ }^{4}$
}

\section{Resumo}

Pogostemon cablin Benth. possui importância econômica devido à produção de óleo essencial utilizado pelas indústrias farmacêuticas e de perfumarias. Dentre os fatores ambientais, a radiação é de extrema importância no desenvolvimento das plantas, onde sua limitação pode comprometer a produção. A aplicação de giberelinas promove o alongamento celular resultando em aumento da altura das plantas. Portanto, o nível de radiação e reguladores vegetais podem influenciar a produção de óleos essenciais devido ao menor acúmulo de biomassa. O objetivo deste trabalho foi avaliar o desenvolvimento vegetativo e produção de óleo essencial de patchouli em condições de sombreamento e após a aplicação de $\mathrm{GA}_{3}$. O delineamento experimental utilizado foi inteiramente casualizado em esquema fatorial 2 x 3 comparando níveis de sombreamento $(0,54$ e $77 \%)$ com e sem aplicação de $\mathrm{GA}_{3}\left(200 \mathrm{mg} \cdot \mathrm{L}^{-1}\right)$. A aplicação de $\mathrm{GA}_{3}$ iniciou-se 30 dias após o plantio das mudas. As plantas apresentaram altura superior em $77 \%$ de sombreamento e com tratamento de $\mathrm{GA}_{3}$ aos 120 dias após o plantio. O número de folhas foi superior em plantas desenvolvidas sem limitação de radiação e após 75 dias da aplicação de $\mathrm{GA}_{3}$. $\mathrm{O}$ número de ramos foi superior em condições não sombreadas e sem aplicação de $\mathrm{GA}_{3}$. A aplicação do regulador vegetal aos 75 dias promoveu maior número de ramos com $54 \%$ de sombreamento. Entretanto, o tratamento com $\mathrm{GA}_{3}$ proporcionou maior produtividade e maior rendimento de óleos essenciais em condições sombreadas. $\mathrm{O} \mathrm{GA}_{3}$ pode ser usado para compensar o efeito negativo de menores níveis de radiação. Isto é de grande interesse no cultivo de patchouli já que a aplicação de $\mathrm{GA}_{3}$ permite o aumento da densidade de plantio e, consequentemente, produção de biomassa e de óleos essenciais. A aplicação de $\mathrm{GA}_{3}$ em condições não sombreadas e com $54 \%$ de sombreamento aumentam as concentrações de patchoulol no óleo essencial.

Palavras-chave: Pogostemon cablin Benth., ácido giberélico, espécie aromática, patchoulol

\begin{abstract}
Pogostemon cablin Benth. has economical importance due to the essential oil which is used in the pharmaceutical and perfume industries. Among the environment factors, the radiation is extremely important for plant development and its limitation can result on decrease of yield. The application of gibberellins promotes the cellular elongation resulting on increase of plant height. Therefore, the radiation level and plant growth regulators can indirectly affect the essential oil production due its accumulation in the biomass. The objective of this work was to evaluate vegetative development and

\footnotetext{
${ }^{1}$ Pesquisadora Dra . em Produção Vegetal pela Universidade Federal do Paraná, UFPR, Curitiba, PR. E-mail: rafaellencstorck@ yahoo.com.br

2 Profs. Drs. do Dept ${ }^{\circ}$ de Fitotecnia, UFPR, Curitiba, PR. E-mail: cicero@ufpr.br; atila.mogor@ufpr.br

${ }^{3}$ Pesquisadora Dr $^{\mathrm{a}}$. do Dept ${ }^{\mathrm{o}}$ de Engenharia Química, UFPR, Curitiba, PR. E-mail: lilian.cocco@ufpr.br

${ }^{4}$ Profs. Drs. do Dept ${ }^{\circ}$ de Engenharia Química, UFPR, Curitiba, PR. E-mail: agnesps@ufpr.br; ciyama@ufpr.br

* Autor para correspondência
} 
essential oil production of patchouli under shading and after $\mathrm{GA}_{3}$ application. The experimental design was completely randomized in a 2 × 3 factorial arrangement comparing shading levels $(0,54$, and $77 \%$ ) with or without $\mathrm{GA}_{3}$ application $\left(200 \mathrm{mg} . \mathrm{L}^{-1}\right)$. The $\mathrm{GA}_{3}$ was applied 30 days after planting. The plants showed a superior height under $77 \%$ of shading and with $\mathrm{GA}_{3}$ at 120 days after planting. The leaf number was superior on plants developed under no radiation limitation and after 75 days of $\mathrm{GA}_{3}$ application. The stem number also was significantly superior under no shading and without $\mathrm{GA}_{3}$. The application of the growth regulator at 75 days also promoted great stem number of branches with $54 \%$ of shading. However, treatment with $\mathrm{GA}_{3}$ increased essential oil yield and productivity and greater yield in $P$. Cablin under shading conditions. The growth regulator can be used to compensate the negative effect of lower radiation levels. This is of great interest for patchouli cultivation as the $\mathrm{GA}_{3}$ application allows to increase plant population and, consequently, biomass and essential oil production.

Key words: Pogostemon cablin Benth., gibberellic acid, aromatic specie, patchoulol

\section{Introdução}

O pacthouli é uma espécie aromática com importância econômica devido à produção de óleos essenciais. O constituinte do óleo essencial da espécie de maior interesse é o patchoulol, que confere além da fragrância amadeirada, propriedades fixadoras aos perfumes. Apesar da grande demanda do mercado consumidor, atualmente o Brasil importa grande volume de óleo essencial de patchouli, onde o desenvolvimento de tecnologias de cultivo para a espécie poderá resultar na produção nacional de óleos essenciais desta espécie.

A radiação afeta a assimilação de carbono e conseqüentemente a produção de óleos essenciais (SANGWAN et al., 2001) já que a síntese destes ocorre a partir de rotas bioquímicas originadas no metabolismo primário.

Em carqueja (Baccharis trimera (Less). D.C) observou-se rendimento de óleos essenciais superior com o aumento dos níveis de radiação aos quais as plantas foram expostas (SILVA et al., 2006). O cultivo de Pogostemon cablin em consórcio com Carica papaya resultou em rendimento de óleo essencial superior quando cultivadas em consórcio comparado ao monocultivo, sugerindo melhor desempenho da espécie sob condições de sombreamento (MUNI-RAN et al., 1999).

Outro fator que pode influenciar o rendimento de óleos essenciais é a aplicação de reguladores vegetais, aumentando ou diminuindo o teor e seus constituintes (SHUKLA; FAROOQI, 1990).
Segundo Sangwan et al. (2001), os reguladores vegetais podem atuar favoravelmente sobre o rendimento e a qualidade dos óleos essenciais em espécies do gênero Mentha, pertencente à família Lamiaceae. Ainda ao comparar o efeito da aplicação de vários reguladores vegetais, observou-se que o $\mathrm{GA}_{3}$ promove aumento no rendimento de óleos essenciais e estimula o crescimento das plantas.

Em Pogostemon cablin, Misra (1995a) testou concentrações de giberelina de 10 a $500 \mathrm{mg} . \mathrm{L}^{-1} \mathrm{e}$ constatou que a aplicação exógena de $250 \mathrm{mg}$. $\mathrm{L}^{-}$ ${ }^{1} \mathrm{de} \mathrm{GA}_{3}$ em condições de sombreamento promoveu maior número de ramos, nós por ramos, folhas verdes, área foliar e rendimento de óleo essencial. A massa fresca foliar, altura das plantas e número de folhas amarelas não foram afetados por estes tratamentos. Entretanto, Misra (1995b) observou em plantas de patchouli não submetidas à ambientes sombreados que a aplicação de $\mathrm{GA}_{3}$ resultou no aumento de altura de plantas e no número de nós por planta.

Este trabalho teve como objetivo avaliar a influência do sombreamento e da aplicação de $\mathrm{GA}_{3}$ no desenvolvimento vegetativo e na produção de óleos essenciais de Pogostemon cablin.

\section{Material e Métodos}

O experimento foi conduzido em condições de casa-de-vegetação durante os meses de setembro de 2006 a janeiro de 2007. As mudas foram obtidas por 
meio de estacas com comprimento médio de $6 \mathrm{~cm}$ e um par de folhas com área foliar reduzida à metade.

O delineamento experimental utilizado foi inteiramente casualizado em arranjo fatorial 2 X 3 com 5 repetições, 5 vasos por parcela e uma planta por vaso. Os vasos apresentavam $17 \mathrm{~cm}$ de altura x $23 \mathrm{~cm}$ de diâmetro e capacidade para aproximadamente $6 \mathrm{Kg}$ de substrato. Para a implantação do experimento amostras do substrato foram coletadas para análise química no Laboratório de Fertilidade do Departamento de Solos da UFPR, o qual apresentou as seguintes características: $\mathrm{CaCl}_{2}$ 4,30; SMP 5,20, $\mathrm{Al}^{+3}\left(\mathrm{cmol}_{\mathrm{c}} / \mathrm{dm}^{3}\right) 3,60 ; \mathrm{H}+\mathrm{Al}^{+3}$ $\left(\mathrm{cmol}_{\mathrm{c}} / \mathrm{dm}^{3}\right) \quad 9,00 ; \mathrm{Ca}^{+2}\left(\mathrm{cmol}_{\mathrm{c}} / \mathrm{dm}^{3}\right) \quad 1,60 ; \mathrm{Mg}^{+2}$ $\left(\mathrm{cmol}_{\mathrm{c}} / \mathrm{dm}^{3}\right) 0,80 ; \mathrm{K}^{+}\left(\mathrm{cmol}_{\mathrm{c}} / \mathrm{dm}^{3}\right) 0,07 ; \mathrm{SB}\left(\mathrm{cmol}_{\mathrm{c}} /\right.$ $\left.\mathrm{dm}^{3}\right)$ 2,47; $\mathrm{T}\left(\mathrm{cmol}_{\mathrm{c}} / \mathrm{dm}^{3}\right)$ 11,47; $\mathrm{P}\left(\mathrm{mg} / \mathrm{dm}^{3}\right)$ 5,70; $\mathrm{C}\left(\mathrm{g} / \mathrm{dm}^{3}\right)$ 41, 4; V(\%) 22; m(\%) 59; Ca/Mg 2,0 e argila $(\mathrm{g} / \mathrm{Kg}) 400,00$. Para a correção do $\mathrm{pH}$ do solo, foi incorporado 8,3 toneladas. ha ${ }^{-1}$ de calcário (100\% PNRT) (RAIJ et al., 1996). Ainda conforme recomendação destes autores, foi incorporado $30 \mathrm{Kg} /$ ha $^{-1}$ de $\mathrm{N}, 130 \mathrm{Kg} /$ ha $^{-1}$ de $\mathrm{P}_{2} \mathrm{O}_{5}$ e $110 \mathrm{Kg} /$ ha ${ }^{-1}$ de $\mathrm{K}_{2} \mathrm{O}$.

Os tratamentos incluíram diferentes níveis de sombreamento (0, 54 e 77\%) utilizando-se Sombrite ${ }^{\circledR}$ e aplicação de Pro Gibb® ${ }^{\circledR}$ 10\% GA (200 mg. L ${ }^{-1}$ ) e testemunha. As aplicações de $\mathrm{GA}_{3}$ foram realizadas com equipamento de pulverização pressurizado por $\mathrm{CO}_{2}$ com vazão e pressão constante (50 Psi), aos 30 e aos 75 dias após a implantação do experimento.

Para determinar com maior precisão os níveis de sombreamento, foram feitas medições dos níveis de radiação utilizando-se o aparelho porômetro, modelo LICOR 1600, em dias ensolarados e nublados.

O desenvolvimento vegetativo do material foi avaliado aos 120 dias após a o plantio, a partir da determinação da altura $(\mathrm{cm})$ considerando-se a altura do ramos principal a partir do substrato, número de ramos e de folhas, massa seca de folhas e de ramos. Para determinação da massa seca de folhas e de ramos, o material vegetal foi mantido em estufa com circulação de ar e temperatura de $65^{\circ} \mathrm{C}$ até atingir peso constante.

A área foliar das plantas de patchouli foi determinada a partir de discos foliares de área conhecida em posições específicas do limbo foliar, evitando-se a nervura central. Foram coletadas folhas apicais, basais e medianas as quais foram pesadas. Retirou-se 50 discos com área conhecida de $0,7854 \mathrm{~cm}^{2}$ de cada folha. Após este procedimento os discos retirados foram pesados e a área foliar foi calculada (FERNANDES, 2000).

Para a extração do óleo essencial utilizou-se $30 \mathrm{~g}$ de tecido foliar com aproximadamente $20 \%$ de umidade para hidrodestilação durante 5 horas em aparelho graduado Clevenger e balão volumétrico de 2L. A quantificação do óleo essencial foi realizada com auxilio de micropipeta e as amostras foram armazenadas em freezer até o momento da análise dos seus constituintes.

As amostras do óleo essencial foram analisadas por meio de cromatografia a gás acoplada à espectometria de massa (CG/EM). Utilizou-se cromatógrafo gasoso Varian, modelo CP 3800 com detector FID (CG_FID), coluna capilar Chrompack de sílica fundida CP-SIL 8 CB, $0.25 \mathrm{~mm}$ de diâmetro interno, $30 \mathrm{~m}$ de comprimento e $0,25 \mu \mathrm{m}$ de filme líquido. Temperatura do injetor: $250{ }^{\circ} \mathrm{C}$, split 1:300, Volume de amostra injetada: 1,0 $\mu \mathrm{l}$. Gás de arraste: hélio $1 \mathrm{~mL} / \mathrm{min}$ constante.Gás de makeup: ar sintético, nitrogênio e hidrogênio.Temperatura do detector FID: $300^{\circ} \mathrm{C}$. Programação de temperatura do forno: temperatura inicial de $50^{\circ} \mathrm{C}$, elevação de temperatura a $180^{\circ} \mathrm{C}$ na razão de $10^{\circ} \mathrm{C}$ permanecendo por 20 minutos; elevação de temperatura a $200^{\circ} \mathrm{C}$ na razão de $20^{\circ} \mathrm{C}$ permanecendo por 1 minuto. Tempo total da corrida: 35 minutos.

As análises de variâncias foram realizadas utilizando-se o programa estatístico ASSISTAT versão 7.4 beta (SILVA; AZEVEDO, 2006). As variâncias dos tratamentos foram testadas quanto à homogeneidade pelo teste de Bartlett e as médias dos tratamentos comparadas pelo teste de Tukey a $5 \%$ de probabilidade. 


\section{Resultados e Discussão}

Houve efeito isolado do fator sombreamento sobre a altura das plantas aos 30 e 75 dias e os níveis de 54 e $77 \%$ de sombreamento promoveram maiores médias. De forma isolada, também aos 75 dias, a aplicação de $\mathrm{GA}_{3}$ promoveu aumento na altura das plantas de patchouli.

Por outro lado, houve interação significativa entre os fatores sombreamento e giberelina para a altura de plantas de Pogostemon cablin aos 120 dias de cultivo em casa-de-vegetação, onde os tratamentos submetidos a 54 e $77 \%$ de sombreamento e na ausência de $\mathrm{GA}_{3}$ proporcionaram a maior altura das plantas. Após a aplicação de $\mathrm{GA}_{3}$, a altura de plantas submetidas a $77 \%$ de sombreamento foi significativamente superior que plantas em ambiente não sombreado e com $54 \%$ de sombreamento (Tabela 1).

Tabela 1. Altura $(\mathrm{cm})$, área foliar $\left(\mathrm{cm}^{2}\right)$, número de folhas e ramos, massa seca de folhas e de ramos de Pogostemon cablin em diferentes níveis de sombreamento e $200 \mathrm{mg} \cdot \mathrm{L}^{-1} \mathrm{GA}_{3}$.

\begin{tabular}{|c|c|c|c|c|c|}
\hline \multirow{2}{*}{ Desenvolvimento Vegetativo } & \multicolumn{4}{|c|}{ Sombreamento $(\%)$} & \multirow{2}{*}{ Média } \\
\hline & & 0 & 54 & 77 & \\
\hline \multirow{2}{*}{ Altura (30 dias) } & Sem $\mathrm{GA}_{3}$ & $6,84 \mathrm{~ns}$ & $10,24 \mathrm{~ns}$ & $9,48 \mathrm{~ns}$ & - \\
\hline & Com $\mathrm{GA}_{3}$ & $7,63 \mathrm{~ns}$ & $9,78 \mathrm{~ns}$ & $10,28 \mathrm{~ns}$ & - \\
\hline Média & & $7,23 \mathrm{~B}$ & $10,01 \mathrm{~A}$ & $9,88 \mathrm{~A}$ & \\
\hline \multirow{2}{*}{ Altura (75 dias) } & Sem GA 3 & $19,20 \mathrm{~ns}$ & $26,86 \mathrm{~ns}$ & $26,80 \mathrm{~ns}$ & $24,28 \mathrm{~b}$ \\
\hline & Com $\mathrm{GA}_{3}$ & $22,61 \mathrm{~ns}$ & $29,56 \mathrm{~ns}$ & $30,06 \mathrm{~ns}$ & 27,41 a \\
\hline Média & & $20,90 \mathrm{~B}$ & $28,21 \mathrm{~A}$ & $28,43 \mathrm{~A}$ & - \\
\hline \multirow{2}{*}{ Altura (120 dias) } & $\mathrm{Sem} \mathrm{GA}_{3}$ & $38,04 \mathrm{Bb}$ & $50,70 \mathrm{Aa}$ & $55,64 \mathrm{Ab}$ & - \\
\hline & Com $\mathrm{GA}_{3}$ & $49,67 \mathrm{Ba}$ & $48,40 \mathrm{Ba}$ & $65,22 \mathrm{Aa}$ & - \\
\hline Média & & - & - & - & \\
\hline \multirow{2}{*}{ Área Foliar } & $\mathrm{Sem} \mathrm{GA}_{3}$ & $42,25 \mathrm{Cb}$ & $80,67 \mathrm{Bb}$ & $108,61 \mathrm{Ab}$ & - \\
\hline & Com $\mathrm{GA}_{3}$ & $58,37 \mathrm{Ca}$ & $96,60 \mathrm{Ba}$ & $132,64 \mathrm{Aa}$ & - \\
\hline Média & & - & - & - & - \\
\hline \multirow{2}{*}{ Número de Folhas (120 dias) } & Sem $\mathrm{GA}_{3}$ & $65,60 \mathrm{~ns}$ & $43,84 \mathrm{~ns}$ & $43,84 \mathrm{~ns}$ & $51,09 \mathrm{~b}$ \\
\hline & Com $\mathrm{GA}_{3}$ & $78,96 \mathrm{~ns}$ & $55,52 \mathrm{~ns}$ & $58,40 \mathrm{~ns}$ & $64,29 \mathrm{a}$ \\
\hline Média & & $72,28 \mathrm{~A}$ & $49,68 \mathrm{~B}$ & $51,12 \mathrm{~B}$ & - \\
\hline \multirow{2}{*}{ Número de Ramos (120 dias) } & Sem GA 3 & $2,92 \mathrm{~ns}$ & $2,68 \mathrm{~ns}$ & $2,28 \mathrm{~ns}$ & - \\
\hline & Com $\mathrm{GA}_{3}$ & $3,16 \mathrm{~ns}$ & $2,64 \mathrm{~ns}$ & $2,52 \mathrm{~ns}$ & - \\
\hline Média & & $3,04 \mathrm{~A}$ & $2,66 \mathrm{~B}$ & $2,40 \mathrm{~B}$ & - \\
\hline
\end{tabular}

Médias com mesmas letras maiúsculas nas linhas e minúsculas nas colunas não diferem estatisticamente pelo Teste de Tukey ao nível de $5 \%$ de probabilidade.

Fonte: Elaboração dos autores.

Entre os diversos fatores ambientais, a radiação é primordial ao desenvolvimento das plantas e alterações nos níveis de radiação aos quais uma espécie está adaptada podem levar a alterações em suas características anatômicas, bioquímicas e conseqüentemente no desenvolvimento (ATROCH et al., 2001). Por outro lado, a aplicação de giberelinas também pode promover o aumento da altura média das plantas, pois estas promovem o alongamento celular (SCAVRONI et al., 2006). Em artemísia (Tanacetun parthenium (L.) Shultz Bip),o aumento do nível de sombreamento também promoveu altura superior, quando comparadas às plantas mantidas em pleno sol (CARVALHO et 
al., 2006). Os resultados obtidos neste experimento estão de acordo com estes autores, pois houve influência do fator sombreamento tanto na presença como na ausência doregulador vegetal.

A área foliar das plantas de Pogostemon cablin foi superior em condição de sombreamento independente da aplicação de giberelina. No entanto, quando aplicada, médias superiores foram obtidas em todas as condições sombreamento (Tabela 1). Aguilera, Ferreira e Cecon (2004) observou em plantas de Siegesbeckia orientalis (botão-de-ouro), que plantas mantidas em $50 \%$ de sombreamento apresentaram área foliar cerca de três vezes maior que plantas cultivadas sem limitação da radiação. Misra (1995 a) relatou que a aplicação de giberelina combinada ao sombreamento promove aumento da área foliar de Pogostemon cablin. Deste modo, os resultados obtidos concordam com estes autores.

Aos 120 dias houve influência dos fatores de forma isolada sobre o número de folhas, onde o tratamento não submetido à condição de sombreamento resultou médias superiores (Tabela 1). Em relação à aplicação do regulador vegetal, este também promoveu aumento no número de folhas aos 120 dias (Tabela 1). Da mesma forma, Bora e Sarma (2006), constaram em plantas de ervilha (Pisum sativum L.) que a aplicação de $\mathrm{GA}_{3}$ promove o aumento do número de folhas quando aplicada em concentrações até $250 \mathrm{mg}$. $\mathrm{L}^{-1}$.

Não houve interação entre os fatores aos 120 dias de cultivo para o número de ramos (Tabela 1). Entretanto, observou-se efeito isolado do fator sombreamento, onde plantas não submetidas às condições de sombreamento apresentaram maior número de ramos (Tabela 1).

Para os teores de massa seca foliar o tratamento não sombreado e sem aplicação de $\mathrm{GA}_{3}$ apresentou a maior média. Entretanto, Alvarenga et al. (2003) observou maiores teores de massa seca foliar em plantas de Croton urucurana Baill. submetidas à limitação de $70 \%$ de radiação quando comparadas as que foram mantidas em condições de radiação plena.
As plantas tratadas ou não com $\mathrm{GA}_{3}$ apresentaram maior massa seca foliar no tratamento não sombreado. Nesta condição, a aplicação de giberelina reduziu o acúmulo de massa seca foliar. Em condição de sombreamento, no entanto, médias significativamente superiores para massa seca foliar foram obtidas com a aplicação do regulador vegetal (Tabela 1). Entretanto, para os teores de massa seca de ramos o tratamento com $54 \%$ de sombreamento foi estatisticamente superior aos demais independente da aplicação do regulador vegetal. Após aplicação da giberelina, foi possível observar em todos os níveis de sombreamento, maiores teores de massa seca de ramos destes tratamentos quando comparados aos mesmos níveis de sombreamento sem aplicação de $\mathrm{GA}_{3}$.

Assim como para maioria das características de desenvolvimento, houve interação significativa entre os fatores no rendimento de óleo essencial. Embora o rendimento de óleo essencial não tenha diferenciado estatisticamente entre os níveis de sombreamento, quando o regulador foi aplicado houve aumento significativo de rendimento em condições de sombreamento (Tabela 2). O uso de reguladores vegetais pode alterar o comportamento de algumas plantas através da sua produtividade bem como do seu metabolismo secundário. Desta forma é possível obter-se um aumento no teor do óleo essencial em algumas espécies (SHUKLA; FAROOQUI, 1990). Misra (1995a, 1995b) relatouem seus trabalhos com patchouli que a concentração de $\mathrm{GA}_{3}$ em $250 \mathrm{mg} . \mathrm{L}^{-1}$ em condições de sombreamento promove maior rendimento de óleo essencial e aumento do número de folhas verdes. Este maior rendimento de óleo essencial nestas condições pode ser explicado pelo aumento do número de folhas, pois as estruturas que armazenam óleo essencial no patchouli estão presentes em maior quantidade nas folhas (HENDERSON et al., 1970). Pode-se observar que resultados semelhantes a este autor foram obtidos nas condições deste experimento mesmo utilizando-se uma concentração inferior de giberelina. 
Tabela 2. Rendimento do óleo essencial ( $\mu \mathrm{L} . \mathrm{g}^{-1}$ de massa seca foliar) e produtividade (L.ha $\left.{ }^{-1}\right)$ de Pogostemoncablinaos 120 dias em diferentes níveis de sombreamento e aplicação de $200 \mathrm{mg} \cdot \mathrm{L}^{-1} \mathrm{GA}_{3}$

\begin{tabular}{cccc}
\hline \multirow{2}{*}{ Rendimento de óleo essencial } & \multicolumn{3}{c}{ Sombreamento (\%) } \\
\cline { 2 - 4 } & $\mathbf{0}$ & $\mathbf{5 4}$ & $\mathbf{7 7}$ \\
\hline $\mathrm{Sem} \mathrm{GA}_{3}$ & $58.26 \mathrm{Aa}$ & $44.04 \mathrm{Ab}$ & $52.96 \mathrm{Ab}$ \\
Com GA $_{3}$ & $51.10 \mathrm{Ba}$ & $86.41 \mathrm{Aa}$ & $85.15 \mathrm{Aa}$ \\
Produtividade óleo essencial & & & \\
Sem GA $_{3}$ & $15,92 \mathrm{Aa}$ & $14,16 \mathrm{Ab}$ & $10,47 \mathrm{Ab}$ \\
Com GA $_{3}$ & $15,99 \mathrm{Ba}$ & $24,95 \mathrm{Aa}$ & $21,84 \mathrm{ABa}$ \\
\hline
\end{tabular}

Médias com mesmas letras maiúsculas nas linhas e minúsculas nas colunas não diferem estatisticamente pelo Teste de Tukey ao nível de 5\% de probabilidade.

Fonte: Elaboração dos autores.

Ao verificar os efeitos da aplicação de $\mathrm{GA}_{3}$ em Artemisia pallens Wall, Farooqi, Deviah e Vasundra (1993) observaram que a concentração 200 mg.L${ }^{1}$ promoveu diferença na produtividade do óleo essencial.

De modo semelhante ao rendimento a alteração nos níveis de sombreamento não promoveu diferença significativa na produtividade do óleo essencial nos tratamentos sem aplicação do regulador vegetal. Por outro lado, as plantas tratadas com regulador vegetal apresentaram maior rendimento de óleo essencial em 54 e $77 \%$ de sombreamento (Tabela 2).

Tanto a síntese do patchoulol quanto do pogostol ocorrem em presença de água (DEGUERRY et al., 2006), isto explicaria a tendência das amostras mantidas em 54\% de sombreamento sem aplicação do regulador vegetal apresentarem maiores concentrações destes compostos (Tabela 3), pois em ambientes onde a radiação não incidiu diretamente, a retenção de água no substrato foi superior. Por outro lado, a tendência apresentada pelos constituintes minoritários, os quais apresentaram maiores concentrações em condições não sombreadas, pode estar relacionada com reação de síntese. Nestas reações, ocorre desprotonização e a molécula de água não é utilizada como substrato, sabendo-se que a síntese destes não é dependente da quebra de uma molécula de água, estes resultados sugerem que a concentração destes constituintes no óleo essencial é diretamente dependente do balanço dos constituintes patchoulol e pogostol, tendo em vista a presença de precursores comuns.

Conforme referência supracitada, o patchoulol e o pogostol são formados em presença de água, entretanto, na ausência de água os mesmos intermediários que dão origem a esses compostos originam o seicheleno e alfa guaieno, respectivamente. Esta tendência também pode ser observada na tabela 3 , onde sem sombreamento houve uma diminuição nos teores de patchoulol e um acréscimo nos teores de seicheleno, a mesma relação pode ser observada entre o pogostol e o alfa guaieno. 
Tabela 3. Constituintes do óleo essencial de Pogostemon cablin aos 120 dias em diferentes níveis de sombreamento e $200 \mathrm{mg} \cdot \mathrm{L}^{-1} \mathrm{GA}_{3}$.

\begin{tabular}{lcccccc}
\hline \multicolumn{1}{c}{ Sombreamento } & $\mathbf{0 \%}$ & $\mathbf{5 4}$ & $\mathbf{7 7 \%}$ & $\mathbf{0 \%}$ & $\mathbf{5 4 \%}$ & $\mathbf{7 7 \%}$ \\
\hline \multicolumn{1}{c}{ Composto (\%) } & \multicolumn{3}{c}{ Sem $\mathbf{G A}_{\mathbf{3}}$} & & \multicolumn{3}{c}{$\mathbf{C o m ~ G A}_{\mathbf{3}}$} \\
\hline Beta patchouleno & 1,29 & 1,10 & 0,99 & 1,07 & 1,08 & 1,16 \\
Cariofileno & 3,00 & 2,34 & 2,5 & 2,34 & 2,27 & 2,64 \\
Alfa guaieno & 8,09 & 6,16 & 6,43 & 6,09 & 6,03 & 6,93 \\
Gama patchouleno & 5,36 & 4,19 & 4,44 & 4,09 & 4,09 & 4,70 \\
Alfa patchouleno & 3,38 & 2,62 & 2,77 & 2,57 & 2,54 & 2,94 \\
Seicheleno & 1,36 & 1,07 & 1,11 & 1,02 & 1,03 & 1,16 \\
Beta guaieno & 0,94 & 0,59 & 0,63 & 0,57 & 0,59 & 0,77 \\
Alfa selineno & 1,77 & 1,36 & 1,52 & 1,34 & 1,30 & 1,52 \\
Alfa bulneseno & 12,10 & 9,28 & 10,65 & 9,28 & 8,80 & 10,43 \\
Pogostol & 4,26 & 4,80 & 4,57 & 4,63 & 4,74 & 4,36 \\
Patchoulol & 49,40 & 55,99 & 54,60 & 57,41 & 57,30 & 54,50 \\
\hline
\end{tabular}

Fonte: Elaboração dos autores.

\section{Conclusões}

O nível de sombreamento e a aplicação de $\mathrm{GA}_{3}$ altera o desenvolvimento vegetativo das plantas de Pogostemon cablin, influenciando a altura, número de folhas, de ramos e área foliar.

O sombreamento combinado à aplicação de giberelina resulta em plantas maiores e com número de folhas reduzido. O número de ramos é superior em ambiente não sombreado, porém, quando o $\mathrm{GA}_{3}$ é aplicado, o aumento no número de ramos é favorecido pelo sombreamento.

Plantas mantidas em ambientes não sombreados apresentam maiores teores de massa seca foliar e menor área foliar.

A aplicação de $\mathrm{GA}_{3}$ promove aumento da área foliar.

O sombreamento e a aplicação de $\mathrm{GA}_{3}$ resulta em maior rendimento e produtividade do óleo essencial de Pogostemoncablin.

\section{Agradecimentos}

Os autores agradecem a Capes pela bolsa

\section{Referências}

AGUILERA, D. B.; FERREIRA, F. A.; CECON, P. R. Crescimento de Siegesbeckiaorientalis sob diferentes condições de luminosidade. Planta Daninha, Viçosa, MG, v. 22, n. 1, p. 43-51, 2004.

ALVARENGA, A. A.; CASTRO, E. M.; CASTRO, E. C. L. J.; MAGALHÃES, M. M. Effects of different light levels on the initial grouwth and photosyntesis of Croton urucurana Baill in southeastern Brazil. Revista Árvore, Viçosa, MG, v. 27, n. 1, p. 53-57, 2003.

ATROCH, E. M. A. C.; SOARES, A. M.; ALVARENGA, A. A.; CASTRO, E. M. Crescimento, teor de clorofilas, distribuição de biomassa e características anatômicas de plantas jovens de Bauhinia forficata Link submetidas à diferentes condições de sombreamento. Ciência $e$ Agrotecnologia, Lavras, v. 25, n. 4, p. 853-862, 2001.

BORA, R. K.; SARMA, C. M. Effect of gibberellic acid and cycocel on growth, yield and protein content of pea. Asian Journal of Plant Sciences, Pakistan, v. 5, n. 2, p. 324-330, 2006.

CARVALHO, L. M.; CASALI, V. W. D.; LISBOA, S. P.; BARBOSA, L. C. A.; CECON, P. R. Crescimento e metabolismo em artemísia em função do nível de irradiância. Horticultura Brasileira, Brasília, v. 24, n. 3, p. 289-294, 2006.

DEGUERRY, F.; PASTORE, L.; WU, S.; CLARK, A.; CHAPPELL, J.; SCHALK, M. The diverse sesquiterpene profile of patchouli, Pogostemon cablin, is correlated with concedida. 
a limited number of sesquiterpene synthases. Archives of Biochemistry and Biophysics, San Diego, v. 454, n. 2, p. 123-136, 2006.

FAROOQI, A. A.; DEVIAH, K. A.; VASUNDRA, M. Effect of some growth regulators and pinching on growth, yield and essential oil content of davana (Artemisia pallens Wall.) Indian Perfumer, Shakarpur Delhi, v. 37, n. 2, p. 19-23, 1993.

FERNANDES, P. D. Análise de crescimento $e$ desenvolvimento vegetal. Campina Grande: UFPB, Departamento de Engenharia Agrícola, 2000. 22 p.

HENDERSON, W.; JAMES, W. H.; HOW, P.; JUDGE, J. Chemical and morphological studies on sites of sesquiterpene accumulation in Pogostemon cablin (Patchouli). Phytochemistry, Oxford, v. 9, n. 6, p. 12191228, 1970.

MISRA, M. Application of gibberellin to Pogostemon cablin plants - growth, photosynthetic pigment content an oil, yield. Biologia Plantarun, Czech Republic, v. 37, n. 4, p. $635-639,1995$ b.

MISRA, M. The effect of gibberellic acid $\left(\mathrm{GA}_{3}\right)$ on the growth, photosynthetic pigment content and oil yield of patchouli (Pogostemon cablin Benth.) plants grown in shade condition. Biologia Plantarun, Czech Republic, v. 17, n. 4, p. 367-370, 1995a.

MUNI-RAM, D. R.; SANTOSH, S.; NAQVI, A. A.; SUSHIL, K. Studies on intercropping of patchouli (Pogostemon patchouli) with papaya (Carica papaya). Journal of Medicinal and Aromatic Plant Science, New Delhi, v. 21, n. 2, p. 358-360, 1999.
RAIJ, B.; CANTARELLA, H.; QUAGGIO, J. A.; FURLANI, A. M. C. Recomendações de adubação e calagem para o Estado de São Paulo. Campinas: Instituto Agronômico e Fundação, 1996. 285 p.

SANGWAN, N. S.; FAROOQI, A. H. A.; SFIABIH, F.; SANGWAN, R. S. Regulation ofessential oil production in plants. Plant Growth Regulation, Dordrescht, v. 34, n. 1, p. 3-21, 2001.

SCAVRONI, J.; VASCONCELLOS, M. C.; VALMORBIDA, J.; FERRI, A. F.; MARQUES, M. O. M.; ONO, E. O.; RODRIGUES, J. D. Rendimento e composição química do óleo essencial de Mentha piperita L. submetida a aplicações de giberilina e citocinina. Revista Brasileira de Plantas Medicinais, Botucatu, v. 8, n. 4, p. 40-43, 2006.

SHUKLA, A.; FAROOQI, A. H. A. E. A review article: ultilization of plant growth regulators in aromatic plant production. Current Research Medicinal \& Aromatic Plants, New Delhi, v. 12, n. 3, p. 152-157, 1990.

SILVA, F. de A. S.; AZEVEDO, C. A. V. de. A new version of the assistat-statistical assistance software. In: WORLD CONGRESS ON COMPUTERS IN AGRICULTURE, 4., 2006, Orlando. Anais... Orlando: American Society of Agricultural Engineers, 2006. p. 393-396.

SILVA, F. G.; PINTO, J. E. P.; CARDOSO. M. G.; NASCIMENTO, E. A.; NELSON, D. L.; SALES, J. de F.; MOL, D. J. de S. Influence of radiation level on plant growth, yield and quality of essential oil in carqueja. Ciência e Agrotecnologia, Lavras, v. 30, n. 1, p. 52-57, 2006. 